\title{
Mycoplasma hominis brain abscess following uterus curettage: a case report
}

\author{
Mouhamad Al Masalma ${ }^{1}$, Michel Drancourt ${ }^{1}$, Henry Dufour ${ }^{2}$, Didier Raoult ${ }^{1}$ and Pierre-Edouard Fournier ${ }^{*}$
}

\begin{abstract}
Introduction: Mycoplasma hominis is mostly known for causing urogenital infections. However, it has rarely been described as an agent of brain abscess.

Case presentation: We describe a case of $M$. hominis brain abscess in a 41-year-old Caucasian woman following uterus curettage. The diagnosis was obtained by $16 \mathrm{~S}$ rDNA amplification, cloning and sequencing from the abscess pus, and confirmed by a specifically designed real-time polymerase chain reaction assay.

Conclusions: Findings from our patient's case suggest that $M$. hominis should be considered as a potential agent of brain abscess, especially following uterine manipulation.
\end{abstract}

\section{Introduction}

Brain abscess is a life-threatening condition resulting from the invasion of brain tissues by microorganisms. Current microbiological documentation, mostly based on direct examination and culture of pus specimens, may underestimate the role of fastidious microorganisms in brain abscess [1]. Among these, Mycoplasma hominis has rarely been reported [2-7]. M. hominis is a fastidious and slowgrowing bacterium, commensal of the genitourinary tract of healthy adults. It mostly causes urogenital infections but may also cause extra-genital infections $[8,9]$. Infections caused by Mycoplasma sp. require specific antibiotic treatment. Lacking a cell wall and folic acid synthesis, they are resistant to antibiotics that target the cell wall or folic acid synthesis [10]. In particular, they are naturally resistant to $\beta$-lactams, which in combination with metronidazole have been recommended as empirical treatment of bacterial brain abscesses [11]. In contrast, $M$. hominis is sensitive to antibiotics that prevent the synthesis of proteins, including tetracyclines [12]. In addition, this bacterium cannot be Gram stained and requires specific culture media. However, molecular methods were successfully used to detect M. hominis from human samples [13].

* Correspondence: pierre-edouard.fournier@univmed.fr

${ }^{1}$ Federation de Microbiologie, Hôpital de la Timone, Marseille, France

Full list of author information is available at the end of the article

\section{Case presentation}

In 2006, a previously healthy, 41-year-old Caucasian pregnant woman was admitted to our hospital with vertigo, severe headache, and left hemiparesis. She had no relevant medical history except two previous normal pregnancies and deliveries. A computed tomography (CT) scan and MRI scan of the brain identified a right fronto-parietal hematoma. The hematoma was surgically drained. Then 10 days later, at 22 weeks of gestation, our patient underwent early spontaneous miscarriage that required uterus curettage, complicated by important metrorrhagia. At three days following the miscarriage, our patient developed obnubilation, and subsequently coma. New cerebral CT and MRI scans revealed a fronto-parietal brain abscess. The abscess was surgically removed, and purulent material was sent to our laboratory. A nosocomial infection being suspected, an intravenous empirical treatment associating vancomycin ( $2 \mathrm{~g} /$ day $)$ and meropenem ( $6 \mathrm{~g} /$ day $)$ was started. Gram staining of the abscess specimen showed numerous polymorphonuclear leukocytes but no microorganism. The specimen was then plated onto $5 \%$ sheep blood agar and chocolate agar (BioMérieux, Marcy L'Etoile, France) and incubated at $37^{\circ} \mathrm{C}$ under aerobic, anaerobic, and microaerophilic conditions for 10 days. Plates were examined daily but no growth was observed. For molecular detection, DNA was extracted from the pus sample using the MagNA Pure LC DNA isolation kit II and the MagNA Pure LC instrument as recommended by the manufacturer (Roche, Meylan, France). Amplification 
and sequencing of the $16 \mathrm{~S}$ rDNA gene were performed using broad range primers as previously described [14]. By comparison with sequences from GenBank, the sequence obtained from the polymerase chain reaction (PCR) product (1,475 bp) was $100 \%$ identical to that of $M$. hominis (GenBank accession number AF443616). As a consequence, the antibiotic treatment was changed to doxycycline, $200 \mathrm{mg} /$ day for 12 weeks. Our patient recovered rapidly. On follow-up, she remained asymptomatic six months after the discontinuation of antibiotics. In order to determine whether the infection was monomicrobial or polymicrobial, the PCR amplicon was subsequently cloned into Escherichia coli using the pGEM-T Easy Vector System (Promega, Charbonnières, France). A total of 100 clones were analyzed by sequencing. Only $16 \mathrm{~S}$ rDNA from $M$. hominis was detected in the 100 clones. The identification of $M$. hominis in our patient and the previously published cases motivated the development of a specific real time-PCR (RT-PCR) assay for this bacterium. 16S rDNA was selected as target. Using the Primer Express software (Applied Biosystems), specific primers and probes were designed as follows: MHMGB16Sd (5'-TGT TAT AAG GGA AGA ACA TTT GCA AT-3'), MHMGB16Sr (5'GCC ATC GCT TTC TGA CAA GG-3') and MHMGB16S probe (FAM-AAA-TGA-TTG-CAG-ACTGAC-MGB) respectively. RT-PCR was performed using a LightCycler (Roche). The PCR mix consisted of $4 \mu \mathrm{L}$ of pus DNA, $10 \mu \mathrm{L}$ of Quantitect Probe PCR Master Mix (Qiagen, Courtaboeuf, France), $20 \mathrm{pM}$ of each primer (Eurogentec, Seraing, Belgium), $0.5 \mu \mathrm{L}$ of Uracil DNA glycosylase (Invitrogen), $0.5 \mu \mathrm{L}$ of $3.125 \mu \mathrm{M}$ MHMGB probe (Applera), and $4 \mu \mathrm{L}$ of water. DNA was amplified using the following cycling parameters: heating at $50^{\circ} \mathrm{C}$ for 2 minutes, and then at $95^{\circ} \mathrm{C}$ for 15 minutes, followed by 50 cycles of a two-stage temperature profile of $95^{\circ} \mathrm{C}$ for one second and $60^{\circ} \mathrm{C}$ for 45 seconds. The specificity of the primers and probes was tested using BLAST http://blast.ncbi. nlm.nih.gov/ and by tentatively amplifying DNA from 24 distinct Mycoplasma species. The system was found to be specific to M. hominis, as no amplification was obtained from any other mycoplasmal or human DNA. For our patient, positive amplification was obtained after $22 \mathrm{PCR}$ cycles. Negative controls remained negative.

\section{Discussion}

M. hominis frequently colonizes the lower genitourinary tract of women [15]. Host predisposing factors such as immunosuppression, malignancy, trauma, and manipulation or surgery of the genitourinary tract are considered as risk factors of extra-genital infections. It was notably demonstrated that blood spread of mycoplasmas may follow urinary tract catheterization or lithiasis [16]. To the best of our knowledge, M. hominis has previously been reported in only six patients as a cause of brain abscess [2-7] (Table 1). In the three female patients, M. hominis infection complicated a traumatic or spontaneous brain hematoma in a context of normal vaginal or cesarean delivery $[2,3,7]$. In the two male adult patients, the $M$. hominis infection complicated a head trauma in the context of urinary tract catheterization $[4,5]$. In female patients, the most likely source of $M$. hominis was the genital tract whereas it was the urinary tract in men. The most recent patient, a three-week-old baby, most likely acquired the $M$. hominis infection from passage through the maternal birth canal [6]. In our patient, we assume that the source of infection was the genital tract, as our patient underwent uterine curettage. It should be noted that in most cases, $M$. hominis superinfected a brain hematoma. By searching the literature for other cases of $M$. hominis infection of hematomas, we found six articles describing patients who had developed infection of abdominal, peri-nephric, thigh or retroperitoneal hematomas following genitourinary invasive procedures [17-22] (Table 2). In an additional patient, infection complicated a peri-hepatic hematoma but the origin of infection was not identified [23]. Therefore, M. hominis appears to have a particular ability for superinfecting hematomas, in particular following genitourinary tract invasive procedures.

In addition, as previously reported [4], bacterial culture and Gram staining results remained negative. $M$. hominis was only detected by PCR. In addition, in an effort to reduce the diagnostic delay, we developed a specific RT-PCR for M. hominis. This test provides a rapid alternative not only to culture but also to broadrange $16 \mathrm{~S}$ rRNA PCR and sequencing detection, and may enable rapid antibiotic treatment adaptation.

Table 1 Epidemioclinical features of previously reported patients with Mycoplasma hominis brain abscess

\begin{tabular}{llll}
\hline Sex/age & Medical history & Identification & Reference \\
\hline M/29 & Traumatic brain hematoma and urinary tract catheterization & Culture & {$[5]$} \\
M/40 & Head trauma and urinary tract catheterization & PCR & {$[4]$} \\
F/22 & Brain hematoma following normal vaginal delivery & Culture & {$[7]$} \\
F/17 & Subdural hematoma following normal full term pregnancy and delivery & Culture & {$[2]$} \\
F/32 & Subdural hematoma following cesarean delivery & Culture & {$[3]$} \\
M/3 weeks & Normal full term pregnancy and delivery & PCR & {$[6]$} \\
\hline
\end{tabular}

$\mathrm{PCR}=$ polymerase chain reaction. 
Table 2 Cases of hematoma (other than brain) infected with Mycoplasma hominis

\begin{tabular}{llll}
\hline Sex/age & Medical history & Identification & Reference \\
\hline F/27 & Abdominal hematoma following cesarean section & Culture & {$[19]$} \\
F/27 & Abdominal hematoma following cesarean section & Culture and PCR & {$[21]$} \\
M/74 & Wound and peri-nephric hematoma following renal transplantation & Culture & {$[22]$} \\
F/18 & Peri-nephric hematoma following renal transplantation & Culture & {$[20]$} \\
F/36 & Thigh hematoma following trauma of pelvis and genitourinary tract & Culture & {$[18]$} \\
M/55 & Peri-hepatic hematoma following liver transplantation & Culture & {$[23]$} \\
M/29 & Retroperitoneal hematoma following pelvis trauma & Culture & {$[17]$} \\
F/69 & Subcutaneous hematoma and respiratory tract infection & Culture & {$[24]$} \\
\hline
\end{tabular}

$\mathrm{PCR}=$ polymerase chain reaction

\section{Conclusions}

Our data suggest that M. hominis should be suspected in patients developing brain abscess following genitourinary tract invasive procedures, notably uterine curettage. To facilitate the detection of this agent, we developed an accurate, sensitive, and specific RT-PCR assay for $M$. hominis that may enable the diagnosis to be obtained within one hour of DNA extraction.

\section{Consent}

Written informed consent was obtained from the patient for publication of this case report and any accompanying images. A copy of the written consent is available for review by the Editor-in-Chief of this journal.

\section{Author details}

${ }^{1}$ Federation de Microbiologie, Hôpital de la Timone, Marseille, France.

${ }^{2}$ Service de Neurochirurgie, Hôpital de la Timone, Marseille, France.

\section{Authors' contributions}

MAM and PEF wrote the manuscript while MD performed the microbiological identification. HD performed the surgical treatment and revised the manuscript. DR corrected the manuscript. All authors read and approved the final manuscript.

\section{Competing interests}

The authors declare that they have no competing interests.

Received: 15 December 2010 Accepted: 3 July 2011

Published: 3 July 2011

\section{References}

1. Al Masalma M, Armougom F, Scheld WM, Dufour H, Roche PH, Drancourt M, Raoult D: The expansion of the microbiological spectrum of brain abscesses with use of multiple $16 \mathrm{~S}$ ribosomal DNA sequencing Clin Infect Dis 2009, 48:1169-1178.

2. Douglas MW, Fisher DA, Lum GD, Roy J: Mycoplasma hominis infection of a subdural haematoma in the peripartum period. Pathology 2003, 35:452-454.

3. Escamilla F, Fernandez MD, Espigares A, Arnal C, Ortega A, Garcia T: Subdural empyema due to Mycoplasma hominis following epidural anesthesia. Rev Neurol 2000, 30:326-328.

4. Kupila L, Rantakokko-Jalava K, Jalava J, Peltonen R, Marttila RJ, Kotilainen E, Kotilainen P: Brain abscess caused by Mycoplasma hominis: a clinically recognizable entity? Eur J Neurol 2006, 13:550-551.

5. Payan DG, Seigal N, Madoff S: Infection of a brain abscess of Mycoplasma hominis. J Clin Microbiol 1981, 14:571-573.
6. Rao RP, Ghanayem NS, Kaufman BA, Kehl KS, Gregg DC, Chusid MJ: Mycoplasma hominis and Ureaplasma species brain abscess in a neonate. Pediatr Infect Dis J 2002, 21:1083-1085.

7. Zheng X, Olson DA, Tully JG, Watson HL, Cassell GH, Gustafson DR, Svien KA, Smith TF: Isolation of Mycoplasma hominis from a brain abscess. J Clin Microbiol 1997, 35:992-994.

8. McMahon DK, Dummer JS, Pasculle AW, Cassell G: Extragenital Mycoplasma hominis infections in adults. Am J Med 1990, 89:275-281.

9. Taylor-Robinson D: Genital mycoplasma infections. Clin Lab Med 1989, 9:501-523

10. McCormack WM: Susceptibility of mycoplasmas to antimicrobial agents: clinical implications. Clin Infect Dis 1993, 17:S200-S201.

11. Mathisen GE, Johnson JP: Brain abscess. Clin Infect Dis 1997, 25:763-779.

12. Myhre EB, Mardh PA: Treatment of extragenital infections caused by Mycoplasma hominis. Sex Transm Dis 1983, 10:382-385.

13. Krijnen MR, Hekker T, Algra J, Wuisman PI, Van Royen BJ: Mycoplasma hominis deep wound infection after neuromuscular scoliosis surgery: the use of real-time polymerase chain reaction (PCR). Eur Spine J 2006 , 15:599-603.

14. Drancourt M, Berger P, Raoult D: Systematic $16 \mathrm{~S}$ rRNA gene sequencing of atypical clinical isolates identified 27 new bacterial species associated with humans. J Clin Microbiol 2004, 42:2197-2202.

15. McCormack WM: Epidemiology of Mycoplasma hominis. Sex Transm Dis 1983, 10:261-262.

16. Simberkoff MS, Toharsky: Mycoplasmemia in adult male patients. JAMA 1976, 236:2522-2524.

17. Burke DS, Madoff S: Infection of a traumatic pelvic hematoma with Mycoplasma hominis. Sex Transm Dis 1978, 5:65-67.

18. Kailath EJ, Hrdy DB: Hematoma infected with Mycoplasma hominis. Sex Transm Dis 1988, 15:114-115.

19. Koshiba H, Koshiba A, Daimon Y, Noguchi T, Iwasaku K, Kitawaki J: Hematoma and abscess formation caused by Mycoplasma hominis following cesarean section. Int J Womens Health 2011, 3:15-18.

20. Legg JM, Titus TT, Chambers I, Wilkinson R, Koerner RJ, Gould FK. Hematoma infection with Mycoplasma hominis following transplant nephrectomy. Clin Microbiol Infect 2000, 6:619-621.

21. Yamaguchi M, Kikuchi A, Ohkusu K, Akashi M, Sasahara J, Takakuwa K, Tanaka K: Abscess formation due to Mycoplasma hominis infection after cesarean section. J Obstet Gynaecol Res 2009, 35:593-596.

22. Orange GV, Jones M, Henderson IS: Wound and perinephric haematomata infection with Mycoplasma hominis in a renal transplant recipient. Nephrol Dial Transplant 1993, 8:1395-1396.

23. Jacobs F, Van de Stadt J, Gelin M, Nonhoff C, Gay F, Adler M, Thys JP: Mycoplasma hominis infection of perihepatic hematomas in a liver transplant recipient. Surgery 1992, 111:98-100.

24. Ridgway EJ, Allen KD: Mycoplasma hominis abscess secondary to respiratory tract infection. J Infect 1994, 29:207-210.

doi:10.1186/1752-1947-5-278

Cite this article as: Al Masalma et al:: Mycoplasma hominis brain abscess following uterus curettage: a case report. Journal of Medical Case Reports 2011 5:278. 